\title{
Getting podiatry research off the ground
}

Andrea Bradley, Jackie Campbell, David Milns, David White, Woody Caan

Few studies have investigated the outcomes of chiropody treatment, especially in a large community sample. There is an increasing need for research in this field. Podiatrists and other professionals allied to medicine need the evidence from research to back up their opinions within health care. Collaboration between clinicians and higher education is critical for success.

This year the combined inspection of the Audit Commission and the King's Fund reported that: 'There has been a decline in rehabilitation over the last

decade. Shortfalls appear to be affecting people with long-term illness or disability disproportionately, with older people experiencing particular disadvantage. Provision outside hospital settings continues to be underdeveloped (Robinson and Turnock, 1998).'

This pattern of underdevelopment and disadvantage is especially worrying in relation to podiatry. Most of the community clientele are over 70 years of age and are seen outside hospital set- tings. There has been a 'poor research base' for chiropodists to draw upon until recently with the development of honours degree graduates (Potter, 1995). Where small scale outcomes research has been undertaken, this has been hampered by the lack of a standardized 'foot disability index' and knowledge of how footcare impacts on activities of daily living (ADL) (Beeson, 1995).

Drastic cuts by the Cambridge and Huntingdon Health Authority (CHHA) in the chiropody con- tract led to the development of a targeted service in Cambridge which was based on an explicit assessment of 'risk'. A systematic clinical 'risk assessment' was prepared to guide the withdrawal of care from elderly clients in 1997. This aimed to be based on evidence from the existing literature and also to use local clinical audit findings and the local health information from across the trust's COMWISE database. The purchasers wished to reinvest this money, cut from community care, into acute services in a trust elsewhere.

Many professionals expressed concern that footcare was to be withdrawn from over 5000 longstanding patients (about half the total case- load) in response to this abrupt cost-saving demand by the CHHA. It was not known whether the low-risk patients would actually emerge unscathed from the loss of their regular chiropody service. However, the podiatry service could not provide any hard evidence as to the possible consequences of service cuts and so the trust's disinvestment programme went ahead.

\section{THE NEED FOR RESEARCH}

Patients did not agree that their treatment was unnecessary. Over 4000 expressed how upset they were through multiple channels including the complaints system and the community health council $(\mathrm{CHC})$; more than the community trust had ever heard before. Roughly a third of the staff posts disappeared and the remaining staff felt a professional responsibility to ensure that the effects of rationing were kept to a minimum. Staff were proud of their capabilities, which they felt were undervalued and they wished to demonstrate the effectiveness of chiropody, including the indirect economic value of preventing acute admissions (e.g. after a fall) or the increased dependency caused by older people going 'off their feet'.

Extensive discussions took place in 1997-98 about the possible measures of outcome which would be appropriate for chiropody practice, including the potential impact on the wider system of care. Many disciplines contributed to this, including local colleagues in 
physiotherapy, occupational therapy, psychology and general practice. Representing service users, the $\mathrm{CHC}$ also backed an evaluation of the service cutbacks. However, in $1997 \mathrm{few}$ of the Cambridge chiropodists had any first-hand experience of research.

\section{GETTING IT TOGETHER}

In such uncharted territory, it was essential to seek specialist research support. The academic researchers of Nene University College proved especially responsive, offering professional, planning and statistical advice in relation to the evaluation. Nene has also supervised the research podiatrist involved and underwritten the first 6 months' research expenses to undertake a pilot study. Without this University/NHS collaboration, the study would have been impossible.

For the evaluation to have a rigorous comparative element, a control group of patients was needed. Hinchingbrooke Trust had very similar clinical practice to Lifespan before the 'disinvestment' of 1997 and pilot work established that their case mix was indistinguishable from the neighbouring trust. There was an incentive for the two community services to cooperate, as CHHA were threatening to make similar cuts in Hinchingbrooke's chiropody department. Needless to say, the health authority had made its decisions in advance of any research evidence of the safety or health effects associated with their first changes (CHHA presentation to the Cambridge CHC, 10 June 1998). The two trusts also sought help from the Society of Chiropodists and Podiatrists and a range of research groups including the Interprofessional Eastern Rehab Group (Caan, 1997) and the AGEnet network of the Medical Research Council.

It was decided that a prospective study of patients judged to be at 'low risk' should be carried out using a matched case control trial design. About 300 eligible cases were identified in the smaller (Huntingdon) service and these were matched individually to people in the larger (Cambridge) service using the following criteria:

- Age

- Sex

- Presence of nail pathology

- Severity of skin pathology.

The median age of subjects in the sample is about 75 years with the majority of the subjects living at home. Patients with severe cognitive problems were excluded from the study but all other co-morbid clinical conditions were included, for example patients with stroke, coronary heart disease, diabetes or oesteoarthritis.

In order to complete repeated (follow-up) observations, a project over 2 years was envisaged. The following comprehensive battery of measures was chosen within a structured protocol (Figure 1).

The source(s) and quantity of podiatric care will be recorded. It is expected there will be differences in levels of foot care between the control and treatment-withdrawal groups, but some individuals may well make alternative arrangements for podiatry, outside of the NHS 'intention to treat' groupings. As well as a comparative analysis of clinical, ADL and QoL ratings and economic estimates of total service costs between the cases in the two services, two additional statistics will be examined. A survival analysis will be conducted to determine how long patients can remain independently in their own home, and a multidimensional cluster analysis to identify the characteristics of those patients who 'do well' with chiropody. 
Ethical approval was gained from the local research ethics committees in both Cambridge and Huntingdon.

\section{GATHERING RESOURCES}

Preliminary work began in 1998 using resources from Nene-University College and NHS 'Culyer' research and development (R\&D) funding. However, more substantial sums were required to see the whole, follow-up project to completion. Historically, few chiropodists have won competitive research funding, but we felt that the national significance of the work merited an attempt. The research team did not put all our eggs in one basket. So far, the project team have been shortlisted for funds from the Welsh Office of Research and Development for Health and Social Care (with a team of collaborators in Wales) and have also prepared a bid to the own Regional NHS R\&D office under their Health Services Research Committee.

These grant applications followed exploratory negotiations with the funding bodies to determine whether they were interested in this area of the care of older people and what sort of funding might become available. Following this invaluable feedback, the proposals were tailored accordingly each bid is distinctive to meet the expectations of the potential funders. Meanwhile the project is accumulating more and more interested collaborators, which is essential for the advisory project board needed to oversee this research, and ultimately for the dissemination of any results. The professional body was approached and private sector podiatrists in this county will be contacted. The researchers are currently revising the regional funding bid following suggestions from the Eastern R\&D office.

\section{GETTING STARTED}

Good planning is essential and a clear project management system helps establish productive teamwork quickly (Caan et al, 1997). Owing to the immediate relevance of the service changes, local podiatrists were highly motivated to take part. The research podiatrist herself was keen to take on this new role, having seen first hand the impact of arbitrary disinvestment on some of her patients who appeared unable to cope without further professional foot care. A key milestone was passed recently when the pilot study demonstrated the feasibility of a larger study, and this has been communicated to the wider circle of collaborators. Progress continues recruiting subjects and making a battery of measurements.

\section{WHERE NEXT?}

The clinicians were interested in quite difficult questions about the impact of podiatry on a whole population of older people, and our ability to target services reliably at 'high/medium risk' groups. Within a year the project have already started the ball rolling, although it awaits grant decisions to know the scale on which it can proceed. Within podiatry many areas of practice are awaiting research and if other practitioners are interested in this area of rehabilitation why not have a go?

\section{LESSONS FOR OTHERS}

A feasible research project will be based on an area that requires evidence in a professional field. There is a need for thorough information gathering in the area wished to be researched. Always bring in collaborators from higher education and service areas. Start with a pilot study. This will high- light problems that otherwise might go unnoticed but also helps considerably when applying for research funding. Put in well crafted and tailored grant applications and obtain advice from wherever possible. Do not give up. 


\section{REFERENCES}

Beeson P (1995) Podiatric perspective: a case study of rheumatoid arthritis and a multidisciplinary approach. $\mathrm{Br} \mathrm{J}$ Ther Rehabil 2: 566-71

Caan W (1997) Collaboration between health-care professions. Br J Ther Rehabil 4: 224-5

Caan W, Wright J, Hampton-Matthews S (1997) Start as you mean to go on: project management for beginners. J Ment Health 6: 467-72

Potter M (1995) CAPAM: an international programme for continuing profes- sional podiatric development. Br J Ther Rehabil 2: 442-8

Robinson J, Turnock S (1998) Investing in Rehabilitation. Kings Fund, London

Figure One - Battery of measures to be used in the study

The pathologies present: nail, skin and ulcers, including a full description and grading scales

The presence and severity of pain, inflammation and infection

Full vascular assessment to include the following: colour and condition of skin, temperature gradient of the lower limb and dorsum of the foot, dorsalis pedis and posterior tibial pulses (presence/absence and noting any irregularities), presence or absence of intermittent claudication, oedema and history of vasospastic disorders

A neurological assessment to be carried out using a $10 \mathrm{~g}$ monofilament on the dorsum, plantar metatarsal, plantar calcaneum and apices of both first toes, and a C128 tuning fork again on the apices of both first toes

Additional clinical observations including any history of falls or injury, relevant medical history and drug therapy

Standardized ratings of the activities of daily living using the Functional Independence Measure and of the quality of life using the MOS Short Form-12

Use of other services including GP call outs, accident and emergency attendances, acute hospital admissions and any transfer to continuing care facilities 